\title{
Search for Exotic Particles at the NA62 Experiment
}

\author{
Maria Brigida Brunetti, Francesco Gonnella *(D), Lorenza Iacobuzio and on behalf of the NA62 \\ Collaboration $t$
}

Edgbaston, University of Birmingham, Birmingham B152TT, UK; maria.brigida.brunetti@cern.ch (M.B.B.); lorenza.iacobuzio@cern.ch (L.I.)

* Correspondence: francesco.gonnella@cern.ch

† The NA62 Collaboration author list: R. Aliberti, F. Ambrosino, R. Ammendola, B. Angelucci, A. Antonelli, G. Anzivino, R. Arcidiacono, M. Barbanera, A. Biagioni, L. Bician, C. Biino, A. Bizzeti, T. Blazek, B. Bloch-Devaux, V. Bonaiuto, M. Boretto, M. Bragadireanu, D. Britton, F. Brizioli, M.B. Brunetti, D. Bryman, F. Bucci, T. Capussela, A. Ceccucci, P. Cenci, V. Cerny, C. Cerri, B. Checcucci, A. Conovaloff, P. Cooper, E. Cortina Gil, M. Corvino, F. Costantini, A. Cotta Ramusino, D. Coward, G. D'Agostini, J. Dainton, P. Dalpiaz, H. Danielsson, N. De Simone, D. Di Filippo, L. Di Lella, N. Doble, B. Dobrich, F. Duval, V. Duk, J. Engelfried, T. Enik, N. Estrada-Tristan, V. Falaleev, R. Fantechi, V. Fascianelli, L. Federici, S. Fedotov, A. Filippi, M. Fiorini, J. Fry, J. Fu, A. Fucci, L. Fulton, E. Gamberini, L. Gatignon, G. Georgiev, S. Ghinescu, A. Gianoli, M. Giorgi, S. Giudici, F. Gonnella, E. Goudzovski, C. Graham, R. Guida, E. Gushchin, F. Hahn, H. Heath, T. Husek, O. Hutanu, D. Hutchcroft, L. Iacobuzio, E. Iacopini, E. Imbergamo, B. Jenninger, K. Kampf, V. Kekelidze, S. Kholodenko, G. Khoriauli, A. Khotyantsev, A. Kleimenova, A. Korotkova, M. Koval, V. Kozhuharov, Z. Kucerova, Y. Kudenko, J. Kunze, V. Kurochka, V.Kurshetsov, G. Lanfranchi, G. Lamanna, G. Latino, P. Laycock, C. Lazzeroni, M. Lenti, G. Lehmann Miotto, E. Leonardi, P. Lichard, L. Litov, R. Lollini, D. Lomidze, A. Lonardo, P. Lubrano, M. Lupi, N. Lurkin, D. Madigozhin, I. Mannelli, G. Mannocchi, A. Mapelli, F. Marchetto, R. Marchevski, S. Martellotti, P. Massarotti, K. Massri, E. Maurice, M. Medvedeva, A. Mefodev, E. Menichetti, E. Migliore, E. Minucci, M. Mirra, M. Misheva, N. Molokanova, M. Moulson, S. Movchan, M. Napolitano, I. Neri, F. Newson, A. Norton, M. Noy, T. Numao, V. Obraztsov, A. Ostankov, S. Padolski, R. Page, V. Palladino, C. Parkinson, E. Pedreschi, M. Pepe, M. Perrin-Terrin, L. Peruzzo, P. Petrov, F. Petrucci, R. Piandani, M. Piccini, J. Pinzino, I. Polenkevich, L. Pontisso, Yu. Potrebenikov, D. Protopopescu, M. Raggi, A. Romano, P. Rubin, G. Ruggiero, V. Ryjov, A. Salamon, C. Santoni, G. Saracino, F. Sargeni, V. Semenov, A. Sergi, A. Shaikhiev, S. Shkarovskiy, D. Soldi, V. Sougonyaev, M. Sozzi, T. Spadaro, F. Spinella, A. Sturgess, J. Swallow, S. Trilov, P. Valente, B. Velghe, S. Venditti, P. Vicini, R. Volpe, M. Vormstein, H. Wahl, R. Wanke, B. Wrona, O. Yushchenko, M. Zamkovsky, A. Zinchenko.

Received: 12 October 2018; Accepted: 4 November 2018; Published: 7 November 2018

Abstract: The NA62 experiment at the CERN Super Proton Synchrotron (SPS) is currently taking data to measure the ultra-rare decay $\mathrm{K}^{+} \rightarrow \pi^{+} \nu \bar{\nu}$. The high-intensity setup, trigger flexibility, detector performance and high-efficiency vetoes make NA62 also suitable for direct searches of long-lived, beyond-the-Standard-Model particles, such as Heavy Neutral Leptons (HNLs), Axion-Like Particles (ALPs) and Dark Photons (DPs); moreover, many rare and forbidden decays are studied at NA62. The status of all these searches is reviewed, together with prospects for future data taking at NA62 after the CERN Long Shutdown 2 (LS2).

Keywords: Heavy Neutral Lepton; Dark Photon; Axion-like Particle; exotic processes; rare decays

\section{Introduction}

NA62 is a fixed target experiment located in the North Area of the CERN Super Proton Synchrotron (SPS). The experiment aims at measuring the Branching Ratio (BR) of the very rare decay $K^{+} \rightarrow \pi^{+} v \bar{v}$ with a 10\% precision in three years of data taking (2015-2018).

The study of this rare decay provides important information on the Cabibbo-Kobayashi-Maskawa (CKM) matrix and plays a fundamental role in searching for the underlying flavour mixing and 
$\mathrm{CP}$-violating mechanisms. Moreover, this mode is unique, since the theoretical prediction of its BR can be computed to an exceptionally high degree of precision, not matched by any other Flavour Changing Neutral Current (FCNC) process involving quarks; this provides an opportunity for new physics searches, if discrepancies between the Standard Model (SM) prediction and the experimental measurement were to be found [1]. Discrepancies are expected in several Physics Beyond the Standard Model (PBSM) scenarios, such as the custodial Randall-Sundrum model [2], Minimal Supersymmetric Standard Model (MSSM) analysis [3], simplified Z/Z' models [4], littlest Higgs with T-parity [5] and Lepton Flavour Universality (LFU) violation models [6]).

\section{The NA62 Experiment}

\subsection{State of the Art}

The Standard Model prediction on $B R\left(K^{+} \rightarrow \pi^{+} \nu \bar{v}\right)$ is [1]:

$$
B R\left(K^{+} \rightarrow \pi^{+} v \bar{v}\right)=(8.4 \pm 1.0) \times 10^{-11} .
$$

E787 and E949, two experiments at Brookhaven National Laboratory (BNL), had already observed the $K^{+} \rightarrow \pi^{+} v \bar{v}$ decay and found its BR to be consistent with the Standard Model prediction [7]:

$$
B R_{\text {meas }}\left(K^{+} \rightarrow \pi^{+} v \bar{v}\right)=\left(17.3_{-10.5}^{+11.5}\right) \times 10^{-11} .
$$

\subsection{Experimental Setup}

At NA62, a 400-GeV/c proton beam extracted from the SPS impinges on a Beryllium target, producing a $75-\mathrm{GeV} / \mathrm{c}$ positively-charged hadron beam, of nominally $750 \mathrm{MHz}$, which contains $6 \%$ kaons, $24 \%$ protons and $70 \%$ pions [1]. With the so-called "decay-in-flight" technique, the $6 \%$ content of positive kaons decays in the 65 m-long Fiducial Volume (FV). The NA62 detectors shown in Figure 1 are briefly described in this section.

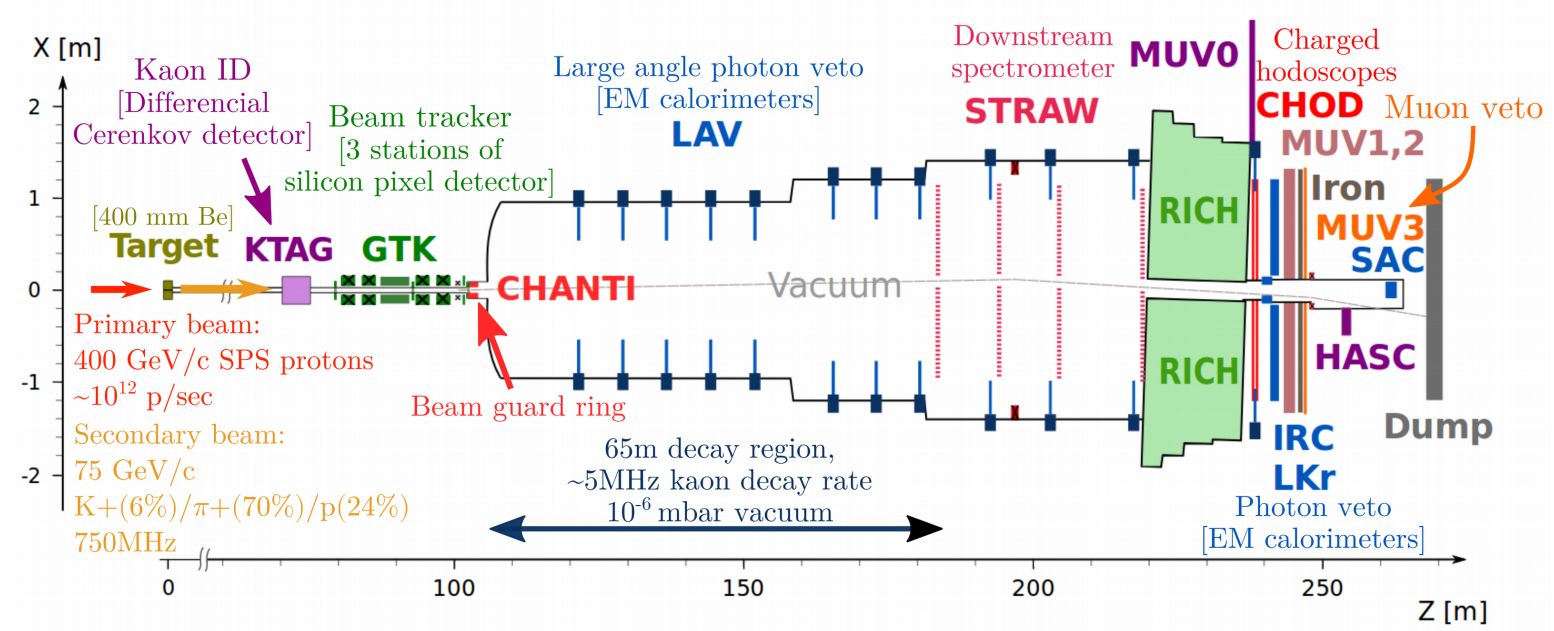

Figure 1. NA62 schematic layout; all the detectors are visible [8].

The particle tracking system is composed of the upstream GigaTracker, used to measure the direction and momentum of the incoming beam, and the downstream Straw spectrometer, used to track the final state charged particles.

The particle identification system is composed of the KTAG, a Cherenkov differential detector used to tag upstream kaons, the RICH, a downstream Cherenkov detector providing pion/muon/electron identification, and the MUVs, a downstream set of hadronic calorimeters and a muon detector.

While searching for rare decays, the ability to reject products of background processes is essential; NA62 uses a hermetic and extremely efficient veto system, in order to reach a signal to background 
ratio of 10:1. The charged particle veto is performed by CHANTI and HASC, whereas the photon veto is composed of LAV, LKrand SAV. Their task is to veto photons, mainly produced by $\pi^{0}$ decays from the $K^{+} \rightarrow \pi^{+} \pi^{0}$ decay (one of the main background processes to $K^{+} \rightarrow \pi^{+} v \bar{v}$ ) and emitted at several angles with respect to the beam axis.

\section{Exotic and Forbidden-Decay Searches at NA62}

Several new physics searches are carried out by the NA62 Collaboration in parallel with the $K^{+} \rightarrow \pi^{+} v \bar{v}$ measurement. These analyses include the search for heavy neutral leptons, axion-like particles, dark photons, lepton-flavour and lepton-number-violating processes. The requirements that the NA62 experimental setup must meet make this experiment suitable for such searches, specifically: a high-intensity setup, a flexible trigger system, high-frequency tracking of beam particles, redundant particle-identification systems and ultra-high-efficiency photon vetoes. In the following sections, the main exotic-search results achieved by NA62 are presented.

\subsection{Heavy Neutral Leptons}

Non-zero masses and mixing of the Standard Model neutrinos are now firmly established; however, many SM extensions have been proposed, involving massive "sterile" neutrinos, or Heavy Neutral Leptons (HNLs), which mix with the ordinary light "active" neutrinos. The Neutrino Minimal Standard Model ( $v \mathrm{MSM})$ postulates three HNLs to explain dark matter and baryon asymmetry of the universe in a way consistent with the results of neutrino oscillation experiments [9]. One of these HNLs, with the expected mass of $\mathrm{O}\left(10 \mathrm{keV} / \mathrm{c}^{2}\right)$, is a dark matter candidate, while the others are expected to have masses of $\mathrm{O}\left(1 \mathrm{GeV} / \mathrm{c}^{2}\right)$. Mixing between $\mathrm{HNLs}$ and active light neutrinos gives rise to HNL production in meson decays, including $K^{+} \rightarrow l^{+} N(l=e, \mu)$. The BR of the latter decay is determined by the HNL mass, $m_{N}$, and the mixing parameter, $\left|U_{l 4}\right|^{2}$, as follows:

$$
B R\left(K^{+} \rightarrow l^{+} N\right)=B R\left(K^{+} \rightarrow l^{+} v\right) \cdot \rho_{l}\left(m_{N}\right) \cdot\left|U_{l 4}\right|^{2}
$$

where $B R\left(K^{+} \rightarrow l^{+} v\right)$ is the measured BR of the SM leptonic decay (including inner bremsstrahlung) and $\rho_{l}\left(m_{N}\right)$ is a kinematic factor [10].

A search for HNL production in $\mathrm{K}^{+} \rightarrow l^{+} N$ decays has been performed at NA62, by analysing data recorded in 2015 during a minimum-bias run. The results are shown in Figures 2 and 3; upper limits on the HNL mixing parameters $\left|U_{e 4}\right|^{2}$ and $\left|U_{\mu 4}\right|^{2}$ in the mass ranges $170-448 \mathrm{MeV} / \mathrm{c}^{2}$ and 250-373 MeV/ $/ \mathrm{c}^{2}$, respectively, have been established at the level between $10^{-7}$ and $10^{-6}$ [10]. This improves on the previous limits from HNL production searches over the whole mass range considered for $\left|U_{e 4}\right|^{2}$ (and extends the mass range in which the limits exist) and above $m_{N}=300 \mathrm{MeV} / \mathrm{c}^{2}$ for $\left|U_{\mu 4}\right|^{2}[10]$.

Analysis of the much larger dataset collected at NA62 between 2016 and 2018 is ongoing; the expected upper limits on $\left|U_{l 4}\right|^{2}$ are expected to improve by at least one order of magnitude.

\subsection{Axion-Like Particles}

With its high beam intensity and the presence of ultra-high efficiency photon vetoes, NA62 has the potential to discover new weakly-coupled particles in the $\mathrm{MeV} / \mathrm{c}^{2}$ to $\mathrm{GeV} / \mathrm{c}^{2}$ mass range, such as the Axion-Like Particles (ALPs), pseudoscalar particles coupled to two photons.

For a high energy proton beam, it is possible to compute the cross-section reliably for the production of ALPs in the $\mathrm{MeV}$ to $\mathrm{GeV}$ range via photon-photon fusion, the so-called Primakoff production [11]. In order to perform this search, NA62 has to be run in dump-mode: two collimators (the TAXes) placed $25 \mathrm{~m}$ after the target, the most upstream one being copper-based, while the other one iron-based, each $1.6 \mathrm{~m}$ long, must be closed and hence act as a beam dump; all the beam remnants and the products of proton-on-target interactions are absorbed by the dump, so that only weakly-coupled 
particles can potentially reach the FV and their decay products detected. Only muons and neutrinos survive the dump and constitute the major source of background for ALPs [11].

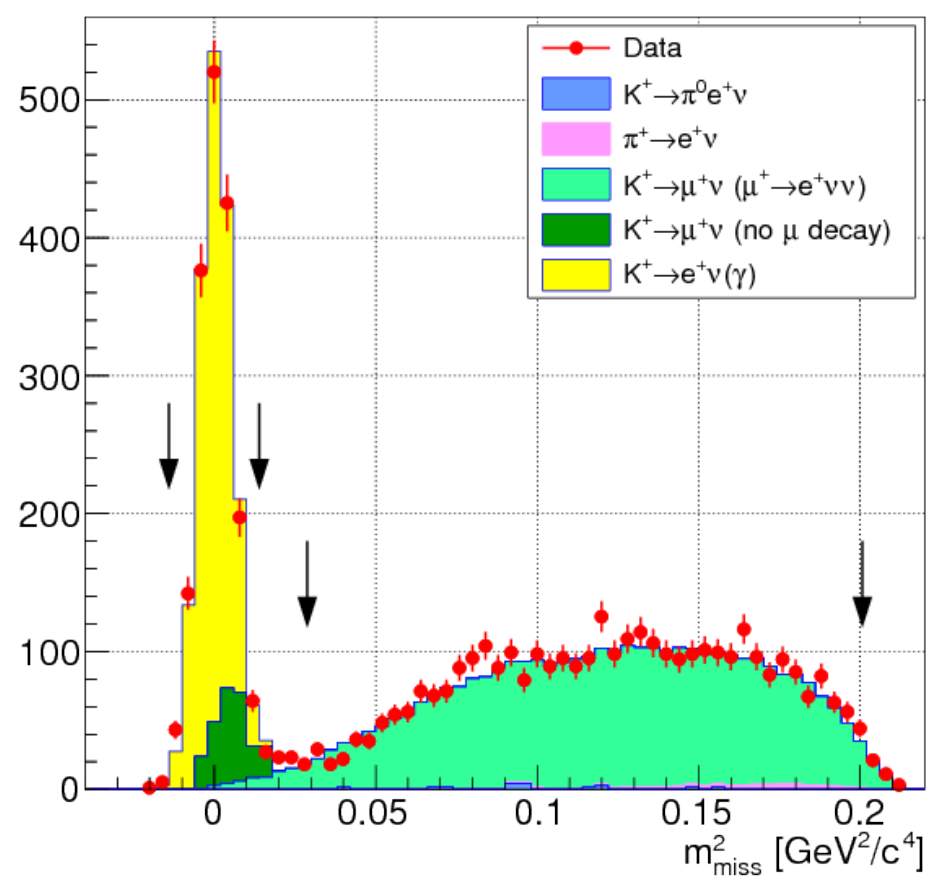

Figure 2. Distributions of the squared missing mass variable $\left(m_{m i s s}^{2}=\left(P_{K}-P_{l}\right)^{2}\right.$, where $P_{K}$ and $P_{l}$ are, respectively, the kaon and lepton four-momenta) for data and simulated events passing the $e^{+}$ selection. Pairs of vertical lines indicate the boundaries of the SM and Heavy Neutral Lepton (HNL) signal regions [10].

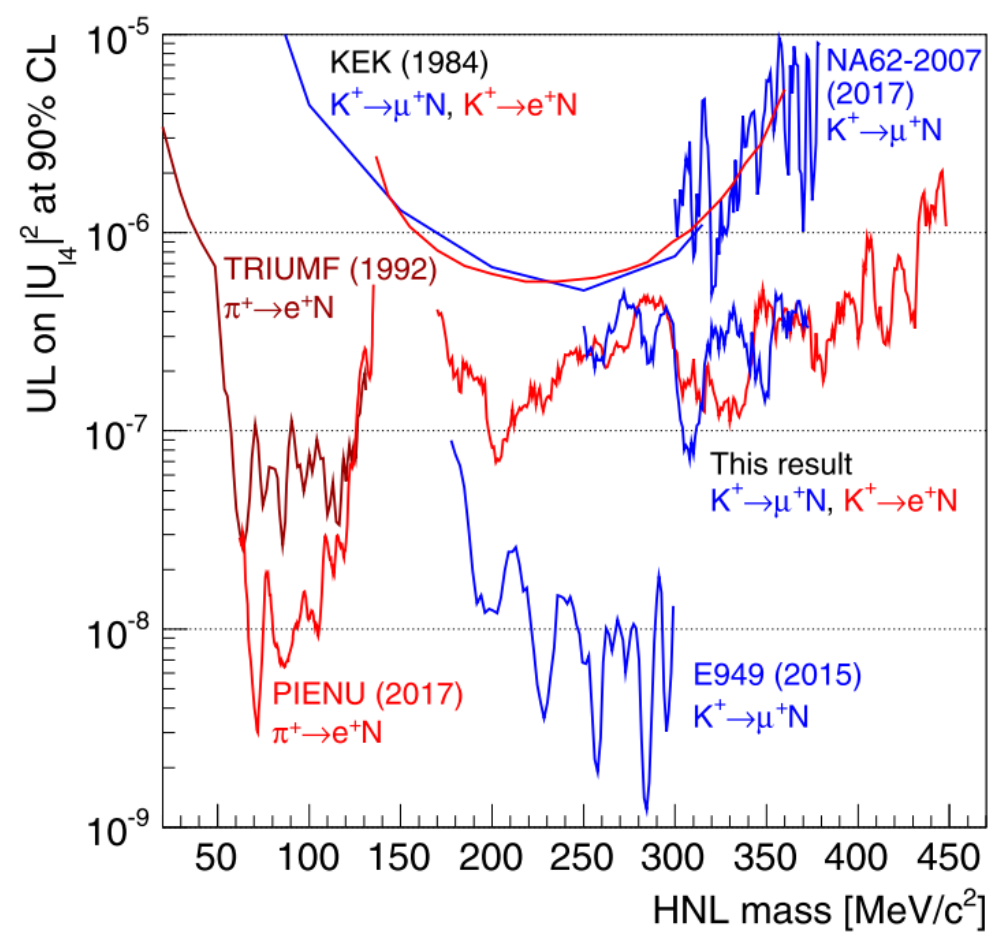

Figure 3. Upper limits at the $90 \%$ Confidence Level (CL) on $\left|U_{l 4}\right|^{2}$ obtained for each assumed HNL mass, compared to the limits established by earlier HNL production searches in $\pi^{+}$decays (TRIUMFand PIENU) and $K^{+}$decays (KEK, E949 and NA62-2007) [10]. 
Primakoff production is particularly interesting for proton beam-dump experiments for two main reasons: the cross-section of the process is proportional to $\sim Z^{2}$; thus, ALPs' production is strongly enhanced for high-Z beam dumps, such as NA62 Copper TAXes. Moreover, ALPs produced via the Primakoff effect have typically a very small transverse momentum; hence, also small detectors placed far from the production point (such as NA62) have a good acceptance [11].

The high-efficiency photon-veto system is suitable to search for the decay of an ALP into two photons $(A \rightarrow \gamma \gamma)$, which is allowed by the coupling $g_{a \gamma}$.

Expected sensitivity projections can be produced for the beam-dump mode, as shown in Figures 4 and 5.

Moreover, a short beam-dump data sample $\left(5 \times 10^{15}\right.$ Protons On Target $\left.(\mathrm{POT})\right)$ has been collected during the 2017 NA62 data taking; its analysis is ongoing.

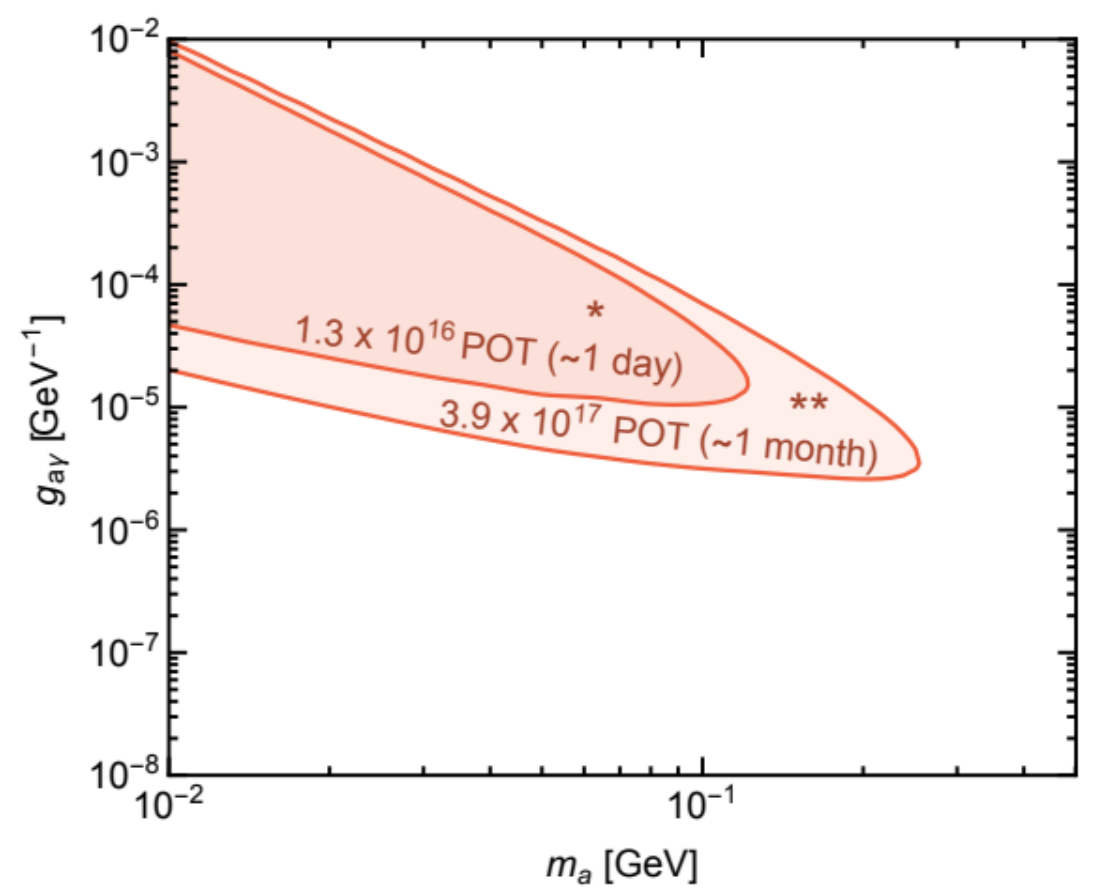

Figure 4. NA62 projected sensitivity, at 90\% CL, in the plane coupling vs. Axion-Like Particle (ALP) mass, under the zero-background hypothesis, for a number of Protons On Target (POT) equivalent to one day of beam-dump mode (marked with ${ }^{*}$ ) and one month (marked with ${ }^{* *}$ ) [11].

\subsection{Dark Photons}

In several hidden-sector models, an extra $\mathrm{U}(1)$ gauge symmetry leads to the presence of an invisible vector boson, $A^{\prime}$, also known as a Dark Photon (DP), which acts as a portal between the SM and the dark sector [12]. The DPs would also provide a possible explanation for the positron excess in cosmic rays and the muon $(g-2)$ measurement [13]. NA62 can search both for visible and invisible DP decays.

DPs decay to invisible via the following chain [14]:

$$
K^{+} \rightarrow \pi^{+} \pi^{0} ; \quad \pi^{0} \rightarrow \gamma A^{\prime} ; \quad A^{\prime} \rightarrow X,
$$

where the DP is not detected and $X$ is an invisible decay product. The main kinematic variable studied for this search is the squared missing mass, defined as:

$$
m_{m i s s}^{2}=\left(P_{K}-P_{\pi}-P_{\gamma}\right)^{2},
$$

where $P_{K}, P_{\pi}$ and $P_{\gamma}$ are, respectively, the four-momenta of the kaon, the charged pion and the photon. 
In the squared missing mass distribution, a peak is expected around $m_{A^{\prime}}$. The main background comes from the SM decay $\pi^{0} \rightarrow \gamma \gamma$, when one photon is lost; however, in that case, the squared missing mass distribution would peak around zero [14].

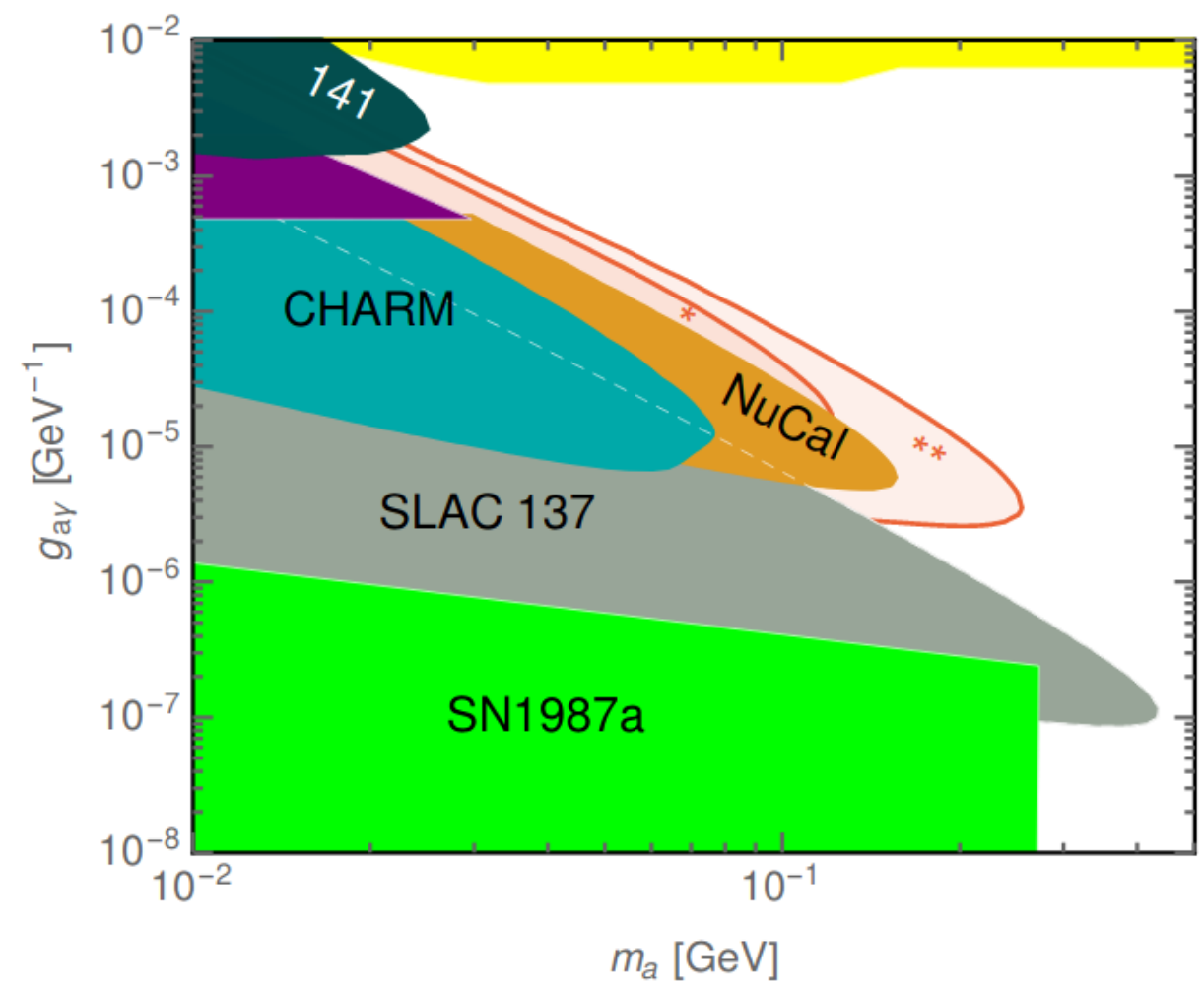

Figure 5. Parameter regions of Figure 4, overlaid with previously performed experiments and astrophysical constraints [11].

The analysis of the partial data sample collected in 2016 allowed setting upper limits on the DP to invisible decays, as shown in Figure 6.

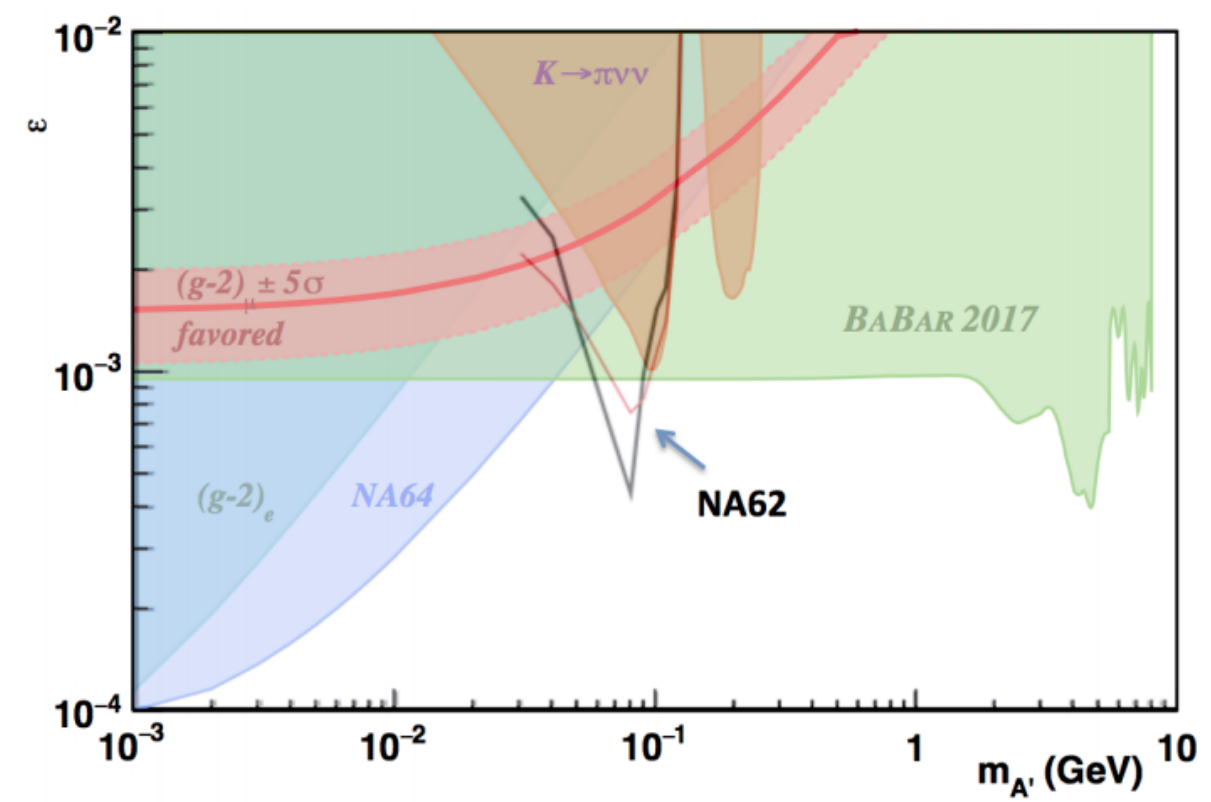

Figure 6. Upper limits (at 90\% CL) in the plane coupling ( $\epsilon$ ) vs. Dark Photon (DP) mass for DP to invisible decays [14]. 
DPs produced in proton interactions with nuclei may decay to visible particles via the two following chains [15]:

$$
p N \rightarrow \pi^{0} X ; \quad \pi^{0} \rightarrow \gamma A^{\prime}, \quad p N \rightarrow X A^{\prime}
$$

with the $A^{\prime}$ decaying in both cases to $l^{+} l^{-}$.

Under the zero-background hypothesis, $10^{18}$ POT are needed to evaluate the expected NA62 sensitivity to DP to visible decays in a region that has not been excluded by past experiments, as shown in Figure 7.

A dataset corresponding to $3.5 \times 10^{17}$ POT has been collected in 2016-2017 at NA62; the analysis of such a data sample is ongoing.

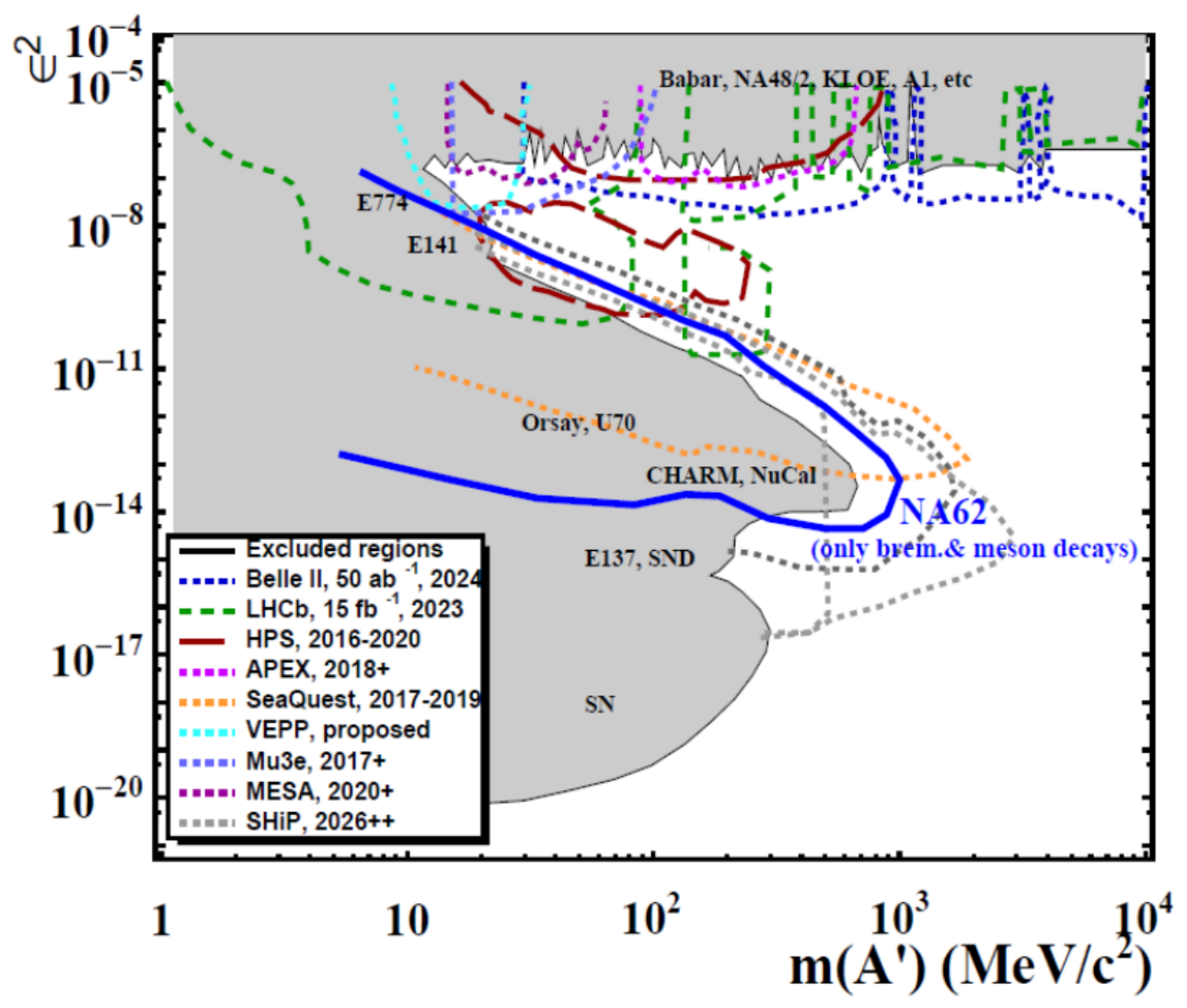

Figure 7. NA62 projected sensitivity (at 90\% CL) in the plane DP-photon coupling ( $\epsilon$ ) vs. DP mass for DP to visible decays [15].

\subsection{Rare and Forbidden Decays}

Measurements of rare kaon and pion decays and searches for decays forbidden in the SM have been performed at NA62 since 2016. A number of analyses are in progress, based on a partial data sample comprising 50\% of the 2016 data and 25\% of the 2017 data.

The status of a search for the Lepton Number-Violating (LNV) decay $\mathrm{K}^{+} \rightarrow \pi^{+} e^{+} e^{-}$with a data sample corresponding to $1.3 \times 10^{11}$ kaon decays in the FV is summarised in Figures 8 and 9 . A background-free signal is observed in the mass region $m_{e e}>140 \mathrm{MeV} / \mathrm{c}^{2}$. The $K^{+} \rightarrow \pi^{+} e^{+} e^{-}$ process is observed for the first time in the kinematic region $m_{e e}<140 \mathrm{MeV} / \mathrm{c}^{2}$, which is possible due to the suppression of the background from the decay chain [15]:

$$
K^{+} \rightarrow \pi^{+} \pi^{0} ; \quad \pi_{\text {Dalitz }}^{0} \rightarrow \gamma e^{+} e^{-} .
$$




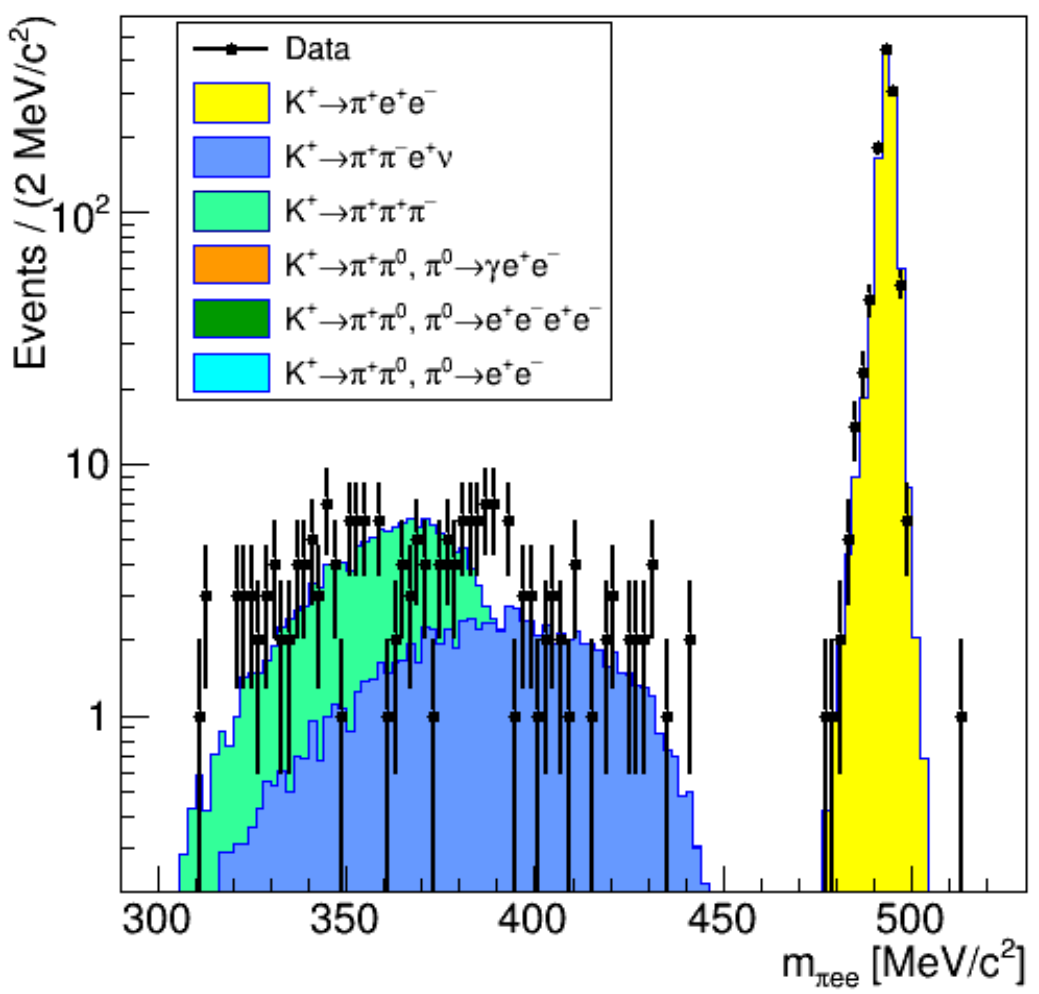

Figure 8. Reconstructed mass distribution of $\mathrm{K}^{+} \rightarrow \pi^{+} e^{+} e^{-}$candidates in the background-free region $m_{e e}>140 \mathrm{MeV} / \mathrm{c}^{2}$; data and MCestimates of various processes are visible [15].

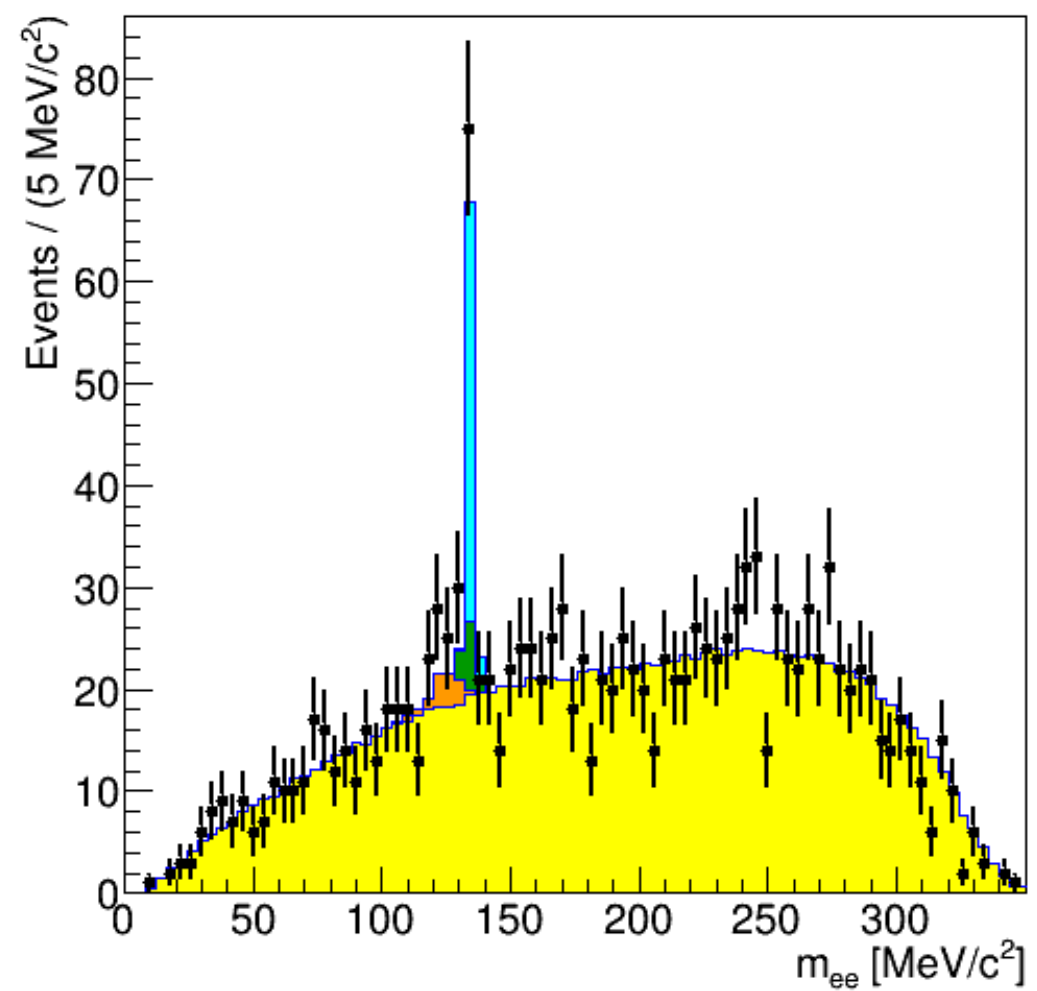

Figure 9. Reconstructed mass distribution of $\mathrm{K}^{+} \rightarrow e^{+} e^{-}$candidates with $488 \mathrm{MeV} / \mathrm{c}^{2}<m_{\pi e e}<$ $500 \mathrm{MeV} / \mathrm{c}^{2}$; data and MC estimates of various processes are visible, together with the NA62 first observation of the rare decay $\pi^{0} \rightarrow e^{+} e^{-}$[15]. 
The status of a search for the LNV decay $K^{+} \rightarrow \pi^{+} \mu^{+} \mu^{-}$with a data sample corresponding to $6.3 \times 10^{11}$ kaon decays in the FV is shown in Figure 10. The world's largest sample is observed, practically with no background [15].

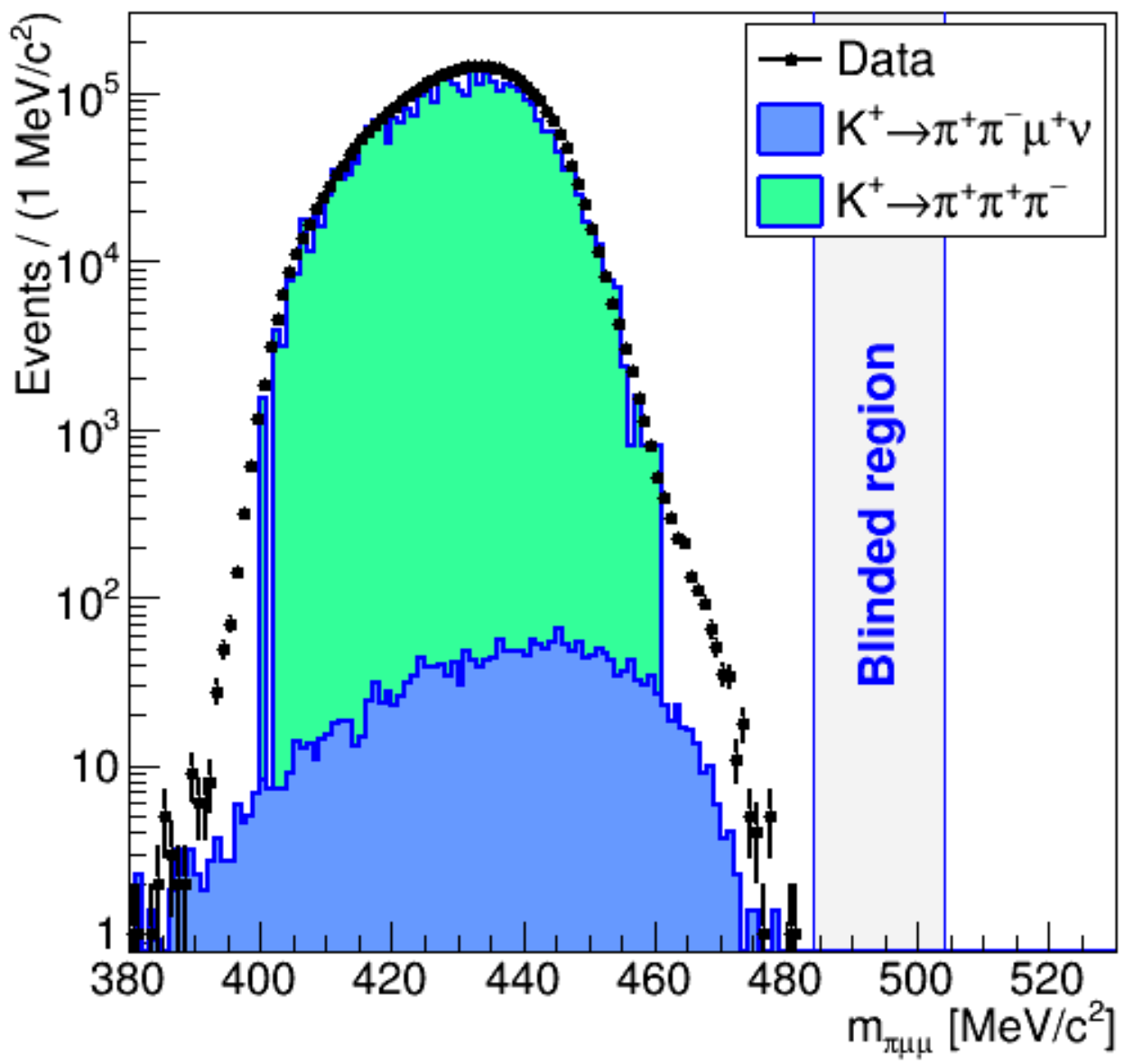

Figure 10. Reconstructed mass spectrum of $K^{+} \rightarrow \pi^{+} \mu^{+} \mu^{-}$candidates: data and MC estimates of potential backgrounds are visible. The description of the tails of the $K^{+} \rightarrow \pi^{+} \pi^{+} \pi^{-}$contribution is limited by simulated statistics; however, data and MC agree within statistical uncertainties [15].

Searches for the LNV decay $\mathrm{K}^{+} \rightarrow \pi^{-} \mu^{+} e^{+}$and the Lepton Flavour Violating (LFV) processes $K^{+} \rightarrow \pi^{+} \mu^{ \pm} e^{\mp}$ with a data sample corresponding to $2.3 \times 10^{11}$ kaon decays in the FV are in progress; none of the searches is limited by background [15]. The single event sensitivities achieved improve over the current limits for the $K^{+} \rightarrow \pi^{-} \mu^{+} e^{+}$and $K^{+} \rightarrow \pi^{+} \mu^{-} e^{+}$modes, but not in the $K^{+} \rightarrow \pi^{+} \mu^{+} e^{-}$ case. NA62 expects to improve on present limits on the decay $\pi^{0} \rightarrow \mu^{ \pm} e^{\mp}$ when analysing the full 2016-2018 dataset [15].

\section{Conclusions}

NA62 is currently taking data to measure the FCNC decay $K^{+} \rightarrow \pi^{+} \nu \bar{v}$ at CERN SPS; the analysis of the 2016-2018 data will improve significantly the state-of-the-art measurement.

Several hidden-sector searches are also performed at NA62, such as searches for HNLs, ALPs and DPs; expected sensitivities have been produced, some of which are in view of a possible beam-dump mode after the CERN LS2. Many rare and forbidden decays, such as LFV and LNV processes, are also studied at NA62, several of which improve the world's single event sensitivities.

Author Contributions: F.G. was involved in NA62 level-0 trigger, M.B.B. participated in the High Level Trigger development and L.I. performed data-analysis. 
Funding: The cost of the experiment and of its auxiliary systems were supported by the funding agencies of the Collaboration Institutes. We are particularly indebted to: F.R.S.-FNRS (Fonds de la Recherche Scientifique-FNRS), Belgium; BMES (Ministry of Education, Youth and Science), Bulgaria; MEYS (Ministry of Education, Youth and Sports), Czech Republic; BMBF (Bundesministerium für Bildung und Forschung) contracts 05H09UM5, 05H12UM5 and 05H15UMCNA, Germany; INFN (Istituto Nazionale di Fisica Nucleare), Italy; MIUR (Ministero dell'Istruzione, dell' Università e della Ricerca), Italy; CONACyT (Consejo Nacional de Ciencia y Tecnología), Mexico; IFA (Institute of Atomic Physics), Romania; INR-RAS (Institute for Nuclear Research of the Russian Academy of Sciences), Moscow, Russia; JINR (Joint Institute for Nuclear Research), Dubna, Russia; NRC (National Research Center) "Kurchatov Institute" and MESRF (Ministry of Education and Science of the Russian Federation), Russia; MESRS (Ministry of Education, Science, Research and Sport), Slovakia; CERN (European Organization for Nuclear Research), Switzerland; STFC (Science and Technology Facilities Council), United Kingdom; NSF (National Science Foundation) Award Number 1506088, U.S.A.; ERC (The European Research Council) "UniversaLepto" advanced grant 268062, "KaonLepton" starting grant 336581, Europe. Individuals have received support from: Charles University (project GA U.K. number 404716), Czech Republic; Ministry of Education, Universities and Research (MIUR “Futuro in ricerca 2012" grant RBFR12JF2Z, Project GAP), Italy; The Royal Society (grants UF100308, UF0758946), United Kingdom; The Science and Technology Facilities Council (Rutherford fellowships ST/J00412X/1, ST/M005798/1), United Kingdom; The European Research Council (ERC “UniversaLepto" advanced grant 268062, ERC “KaonLepton" starting grant 336581).

Conflicts of Interest: The authors declare no conflicts of interest.

\section{References}

1. NA62 Collaboration. Proposal to Measure the Rare Decay $K^{+} \rightarrow \pi^{+} v \bar{v}$ at the CERN SPS. Available online: https://na62.web.cern.ch/na62/Documents/Proposal\%20spsc-2005-013.pdf (accessed on 10 October 2018).

2. Blanke, M.; Burasa, A.J.; Dulinga, B.; Gemmlera, K.; Gori, S. Rare K and B decays in a warped extra dimension with custodial protection. J. High Energy Phys. 2009, 2009, 108. [CrossRef]

3. Isidori, G.; Mescia, F.; Paradisi, P.; Smith, C.; Trine, S. Exploring the flavour structure of the MSSM with rare K decays. J. High Energy Phys. 2006, 2006, 064. [CrossRef]

4. Buras, A.; Buttazzo, D.; Knegjens, R. $K \rightarrow \pi v \bar{v} \varepsilon^{\prime} / \varepsilon$ and in simplified new physics models. J. High Energy Phys. 2015, 2015, 166. [CrossRef]

5. Blanke, M.; Buras, A.J.; Recksiegel, S. Quark flavour observables in the Littlest Higgs model with T-parity after LHC Run 1. Eur. Phys. J. C 2016, 4, 182. [CrossRef] [PubMed]

6. Bordone, M.; Buttazzo, D.; Isidori, G.; Monnard, J. Probing lepton-flavour universality with $K \rightarrow \pi v \bar{v}$ decays. Eur. Phys. J. C 2017, 9, 618. [CrossRef]

7. ATLAS Collaboration. Search for new phenomena in the dijet mass distribution using pp collision data at $\sqrt{s}=8 \mathrm{TeV}$ with the ATLAS detector. Phys. Rev. D 2015, 91, 052007. [CrossRef]

8. NA62 Collaboration. The beam and detector of the NA62 experiment at CERN. J. Instrum. 2017, 12, 05025.

9. Gorbunov, D.; Shaposhnikov, M. How to find neutral leptons of the vMSM? J. High Energy Phys. 2007, $2007,015$. [CrossRef]

10. NA62 Collaboration. Search for heavy neutral lepton production in K+ decays. Phys. Lett. B 2018, 778, 137-145. [CrossRef]

11. Döbrich, B.; Jaeckel, J.; Kahlhoefer, F.; Ringwald, A.; Schmidt-Hoberg, K. ALPtraum: ALP production in proton beam dump experiments. J. High Energy Phys. 2016, 2016, 018. [CrossRef]

12. Holdom, B. Two U (1)'s and $\epsilon$ charge shifts. Phys. Lett. B 1986, 166, 196-198. [CrossRef]

13. Pospelov, M. Secluded U (1) below the weak scale. Phys. Rev. D 2009, 80, 095002. [CrossRef]

14. NA62 Collaboration. INDEX SPSC DOCUMENTS. 2017. Available online: http://cds.cern.ch/record/ 2299464/files/Index_SPSC_2017.pdf (accessed on 10 October 2018).

15. NA62 Collaboration. NA62 Status Report to the CERN SPSC. 2018. Available online: http:/ / cds.cern.ch/ record/2312430 (accessed on 10 October 2018).

(C) 2018 by the authors. Licensee MDPI, Basel, Switzerland. This article is an open access article distributed under the terms and conditions of the Creative Commons Attribution (CC BY) license (http://creativecommons.org/licenses/by/4.0/). 